\title{
Multistability of a coherent spin ensemble in a semiconductor microcavity
}

\author{
T. K. Paraïso ${ }^{1 \star}$, M. Wouters ${ }^{2}$, Y. Léger ${ }^{1}$, F. Morier-Genoud ${ }^{1}$ and B. Deveaud-Plédran ${ }^{1}$
}

Coherent manipulation of spin ensembles is a key issue in the development of spintronics. In particular, multivalued spin switching may lead to new schemes of logic gating and memories. This phenomenon has been studied with atom vapours 30 years ago, but is still awaited in the solid state. Here, we demonstrate spin multistability with microcavity polaritons in a trap. Owing to the spinor nature of these light-matter quasiparticles and to the anisotropy of their interactions, we can optically control the spin state of a single confined level by tuning the excitation power, frequency and polarization. First, we realize high-efficiency power-dependent polarization switching. Then, at constant excitation power, we evidence polarization hysteresis and determine the conditions for realizing multivalued spin switching. Finally, we demonstrate an unexpected regime, where our system behaves as a high-contrast spin trigger. These results open new pathways to the development of advanced spintronics devices and to the realization of multivalued logic circuits.

S pin manipulation is the object of an intense research activity in a great variety of solid-state systems ${ }^{1-3}$. Owing to significant advances in tunability and miniaturization, semiconductor nanostructures have turned into ideal laboratories to address spintronics challenges ${ }^{4}$. In this respect, microcavity polaritons hold great potential ${ }^{5,6}$. Arising from the normal-mode coupling between cavity photons and quantum-well excitons, polaritons behave as bosons and possess unique coherence properties that have led to the demonstration of Bose-Einstein condensation and superfluidity $^{7-9}$. A great advantage of polaritons is the one-to-one correspondence between the polariton spin and the polarization of the emitted light. This allowed the observations of the optical spin Hall effect ${ }^{10}$, or of half-quantum vortices ${ }^{11}$, which have shown that polaritons exhibit remarkable spin carrier properties. Finally, recent realizations of optical bistability ${ }^{12,13}$ and electrical injection in polariton diodes ${ }^{14}$ allow the implementation of low-power polaritronic devices working at room temperature ${ }^{15,16}$.

Spin multistability refers to the possibility for a system to present three or more stable spin states for a given excitation condition. It requires precise control of coherence and interactions and is therefore difficult to realize. The only successful studies of multistability with a spinor system were carried out with atomic vapours 30 years ago ${ }^{17,18}$. Its demonstration in the solid state would clearly lead to new schemes of spin-based logic devices ${ }^{19,20}$. Microcavity polaritons were recently predicted to be promising candidates to explore spin multistability ${ }^{21}$. This phenomenon rapidly emerged as an innovative solution for the design of spin memory elements ${ }^{22}$, and for the realization of logic gates based on the selective transport of spin-polarized polaritons ${ }^{23,24}$. Such developments first require an experimental demonstration of spin multistability in a patterned environment and a characterization of the interactions' strengths.

We realize spin multistability in a semiconductor microcavity where polaritons are localized in cylindrical structures (mesas) of $3 \mu \mathrm{m}$ diameter ${ }^{25}$ (see Fig. 1a,b). The typical polariton spectrum obtained in such mesas is characterized by the coexistence of localized (zero-dimensional, 0D) and delocalized (two-dimensional, 2D) states for both the upper and lower polariton branches. Owing to the discrete energy spectrum of 0D states, the experiments are carried out with a single polariton energy level. In this work, we focus on the ground state of the lower polariton. We excite the mesa at normal incidence $(k=0)$ using a tunable, single-frequency, continuous-wave laser (see the Methods section). We manipulate the spin state of the polariton level by accurately tuning the excitation power, frequency and polarization. Note that the laser energy detuning $\Delta$ with respect to the polariton ground state is kept smaller than the energy difference between the first excited state and the ground state. The excitation polarization is tuned between circular, elliptical or linear using a quarter-wave plate. We characterize the complete polariton spin state and follow the multistability trajectory in the Bloch sphere.

To control the new phenomenology of polariton bistability in the presence of spinor interactions, it is important to understand the influence of the different excitation parameters. We scanned the excitation power for different values of the excitation circular polarization degree $\rho_{\text {pump }}$, and resolved the emission in the circular $(\sigma+, \sigma-)$ basis. In this basis, one can first think about two uncoupled polariton spin-polarizations, $\sigma+$ and $\sigma-$, excited independently by the laser. The respective effective excitation powers $P_{\sigma \pm}$ of $\sigma+$ and $\sigma-$ polaritons depend on both the total excitation power $P_{\text {pump }}$ and the circular polarization degree $\rho_{\text {pump }}$

$$
P_{\sigma \pm}=P_{\text {pump }} \frac{1 \pm \rho_{\text {pump }}}{2}
$$

The emission circular polarization degree is given by

$$
\rho_{\mathrm{C}}=\frac{I_{\sigma+}-I_{\sigma-}}{I_{\sigma+}+I_{\sigma-}}
$$

where $I_{\sigma \pm}$ are the intensities detected for $\sigma \pm$ polaritons.

Under circularly polarized excitation, a single spin-polarization is excited and the emission intensity exhibits conventional bistability ${ }^{12,21}$. The schematic diagrams in Fig. 2a show that a much richer behaviour is obtained under elliptical or linear polarization. The reason is that the strengths of interactions between polaritons of the same $\left(\alpha_{1}\right)$ and opposite $\left(\alpha_{2}\right)$ spin are not equal. Therefore, both spin populations experience different blueshifts: $\Delta \epsilon_{\uparrow}=\alpha_{1} n_{\uparrow}+\alpha_{2} n_{\downarrow}$

\footnotetext{
${ }^{1}$ Laboratory of Quantum Optoelectronics, EPFL, CH-1015, Lausanne, Switzerland, ${ }^{2}$ Institute of Theoretical Physics, EPFL, CH-1015, Lausanne, Switzerland. *e-mail: taofiq.paraiso@epfl.ch.
} 
a

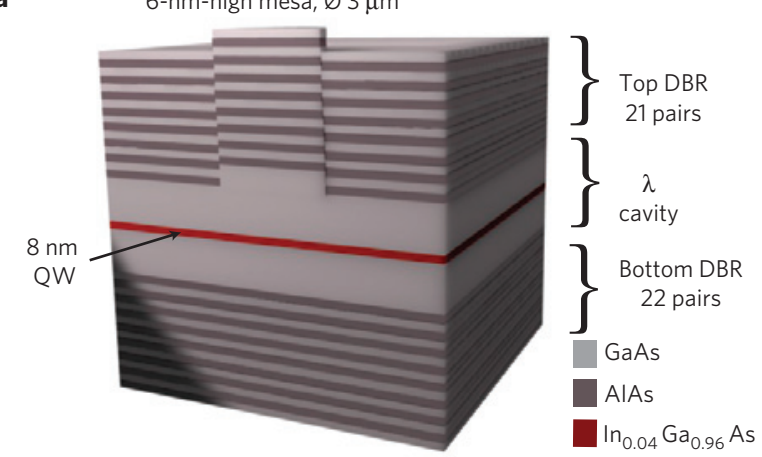

c

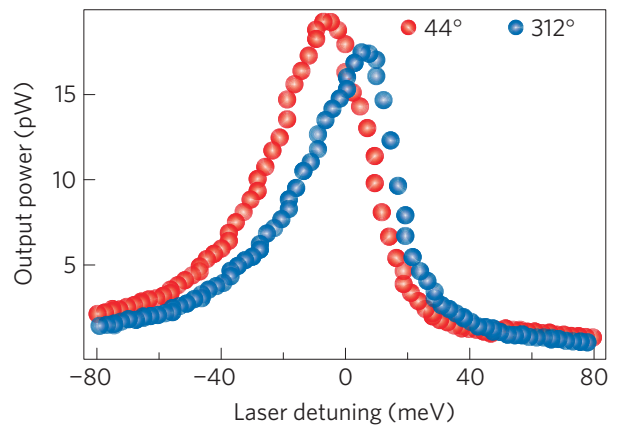

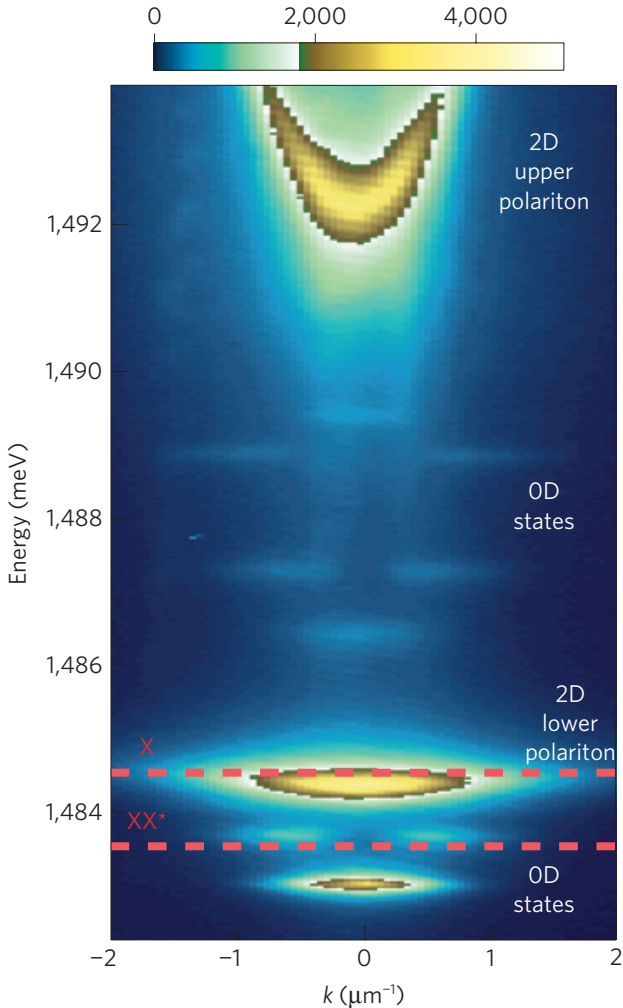

Figure 1 | Polariton lateral confinement. a, Our sample is a semiconductor III-V microcavity in which we engineered local cylindrical extensions of the cavity spacer (mesas). The 6-nm-high mesas trap polaritons through their photonic component. We measured Rabi splittings of 3.15 meV and 3.35 meV in the planar region and in the mesas, respectively. QW: quantum well; DBR: distributed Bragg reflector. $\mathbf{b}$, Polariton spectrum of a 3- $\mu \mathrm{m}$-diameter mesa measured under non-resonant excitation. One clearly distinguishes the continuous spectrum of 2D polariton states and the discrete spectrum of OD polariton states. At slightly positive cavity detuning $(\delta=0.15 \mathrm{meV})$ for the ground polariton state, the spacing between the ground state and the first excited state is more than $0.6 \mathrm{meV}$. We show the estimated biexciton resonance $\left(X X^{\star}\right)$ at $1 \mathrm{meV}$ below the quantum-well excitons' $(X)$ energy,

$1,484.57 \mathrm{meV}$. c, Characterization of the linear polarization splitting. We measure a splitting of $15-20 \mu \mathrm{eV}$ for orientations of the linear polarization directed along crystallographic axes at about $\pm 45^{\circ}$ with respect to the horizontal polarization axis (see the Methods section).

and $\Delta \epsilon_{\downarrow}=\alpha_{1} n_{\downarrow}+\alpha_{2} n_{\uparrow}$ for spin-up $(\sigma+)$ and spin-down $(\sigma-)$ polaritons, respectively.

The experimental data are plotted in Fig. 2b-e. For this experiment, the detuning of the polariton ground state with respect to the exciton energy is $\delta^{\prime}=-1.47 \mathrm{meV}$ and the laser detuning with respect to the polariton state is $\Delta=0.49 \mathrm{meV}$. For nearly $\sigma+$ excitation $\left(P_{\sigma+} \gg P_{\sigma-}\right)$, the coherent emission is dominated by $\sigma+$ polaritons (see Fig. $2 \mathrm{~b}$ ). The corresponding emission polarization degree $\rho_{\mathrm{C}}$ (see equation (2)) is close to 1 during the whole power scan. When $\rho_{\text {pump }}$ is decreased, the proportion of $\sigma-$ polaritons increases and becomes sufficient to cause a jump to the upper intensity branch within the range of the power scan (see Fig. 2c). The abrupt changes in population ratio cause large variations of $\rho_{\mathrm{C}}$ and hence polarization conversion. As $\rho_{\text {pump }}$ approaches 0 ( $P_{\sigma+} \gtrsim P_{\sigma-}$; see Fig. $\left.2 \mathrm{~d}\right)$, simultaneous jumps to the upper branch are observed for both polarizations, indicating repulsive interactions between $\sigma+$ and $\sigma$ - polaritons. The $\sigma$ - hysteresis widens, but remains narrower than the $\sigma+$ hysteresis, suggesting important nonlinear losses in the presence of polaritons with antiparallel spins. At the lower threshold of $\sigma-$ polaritons, the emission polarization is then converted to fully circular.

Polarization conversion is most impressive in Fig. 2e, where under linearly polarized excitation $\rho_{\text {pump }}=0\left(P_{\sigma+}=P_{\sigma-}\right)$, the emission polarization can be reversibly switched from linear $\rho_{\mathrm{C}}=0$ to circular $\rho_{\mathrm{C}}=1$ within very small variations of the excitation power (inset).

The excitation powers are indicated for a $20-\mu \mathrm{m}$-diameter spot. The nominal excitation powers for the $3-\mu \mathrm{m}$-diameter mesa are thus 50 times lower. In Fig. 2b, the excitation power needed to jump on the upper branch is $1.75 \mathrm{~mW}$, which corresponds to an effective power density of $500 \mathrm{~W} \mathrm{~cm}^{-2}$ for the mesa. Polarization switching cycles could be realized within variations of the power density of less than $50 \mathrm{~W} \mathrm{~cm}^{-2}$. Our micrometre-sized trapping structures are therefore the state-of-the-art structures for lowpower polarization-switching ${ }^{24}$.

We analyse our data with a two-mode model based on the spinor Gross-Pitaevskii equation. We qualitatively reproduce the experimentally observed trends and extract information about the relevant interactions in the system (see Supplementary Information). Our model accounts for anisotropic spinor interactions $\left(\alpha_{1}\right.$ and $\left.\alpha_{2}\right)$, polariton linewidth $\gamma$ and linear polarization splitting $\epsilon_{\text {lin. }}$. The last two parameters were directly measured experimentally (see Fig. 1b,c). Moreover, losses resulting from biexciton generation or spin-flip scattering producing dark excitons are described by a nonlinear contribution to the decay rate $\beta$. The two coupled equations for the field amplitudes $\psi_{ \pm}$in the $(\sigma+, \sigma-)$ basis read

$$
\begin{aligned}
i \frac{\mathrm{d}}{\mathrm{d} t} \psi_{ \pm} & =\left[-\Delta-\frac{i}{2}\left(\gamma+\beta\left|\psi_{\mp}\right|^{2}\right)+\alpha_{1}\left|\psi_{ \pm}\right|^{2}+\alpha_{2}\left|\psi_{\mp}\right|^{2}\right] \psi_{ \pm} \\
& +\frac{\epsilon_{\text {lin }} \psi_{\mp}}{2}+F_{ \pm}
\end{aligned}
$$

where $F_{ \pm}$is the excitation field.

A first issue concerns the sign of the interactions between polaritons of opposite spins $\alpha_{2}$. We have experimental evidence for the manifestation of repulsive interactions between polaritons 

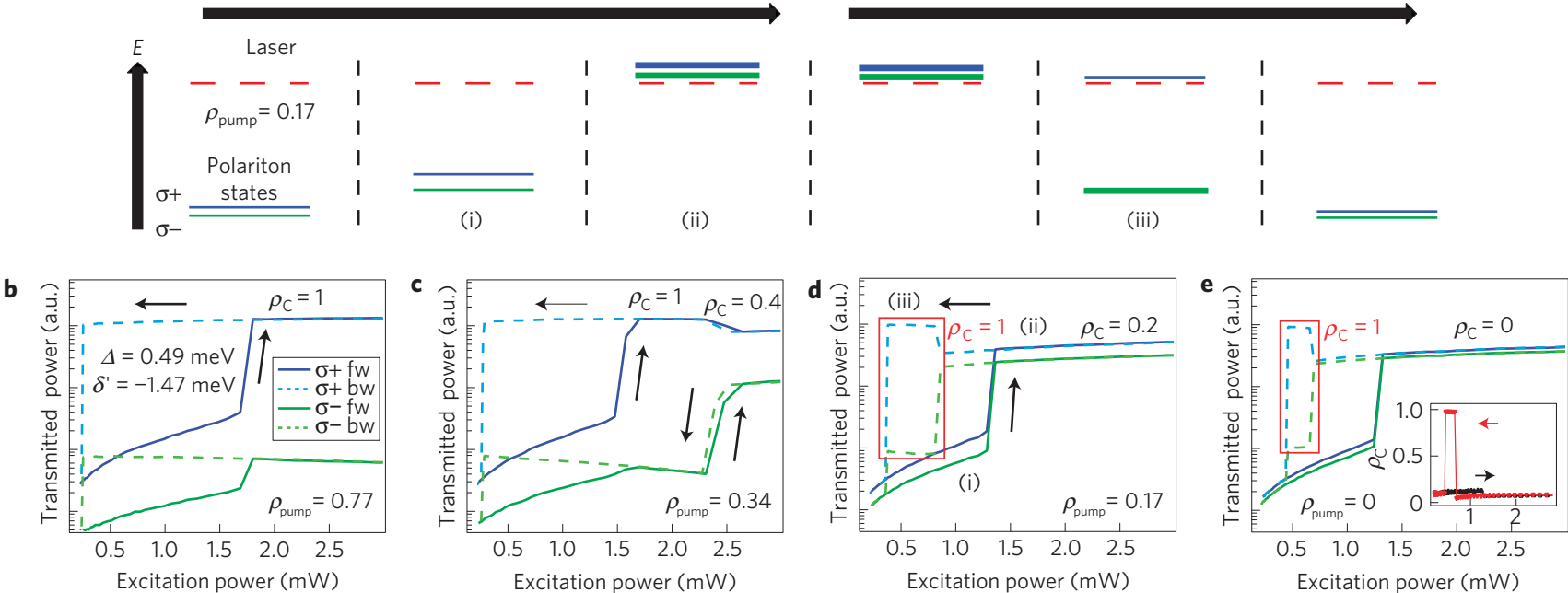

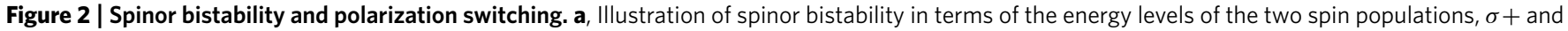

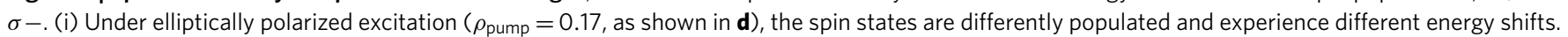
(ii) Small repulsive interactions between $\sigma+$ and $\sigma-$ polaritons cause simultaneous jumps to the upper branch (see $\alpha_{2}$ in equation (3)). (iii) As a result of nonlinear losses, the linewidth of one spin state (line thickness) mainly depends on the population in the other spin state (see $\beta$ in equation ( 3 )). This favours the jumps to the lower branch to be independent, allowing for polarization switching. b-e, Experiment (logarithmic scale). The excitation power is

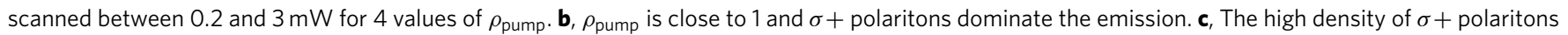
inhibits the $\sigma$ - hysteresis. d,e, $\rho_{\text {pump }}=0.17$ and 0 . We obtain high-contrast linear-to-circular polarization conversion (red rectangles). The inset in $\mathbf{e}$ shows corresponding polarization switching from $\rho_{\mathrm{C}}=0$ to $\rho_{\mathrm{C}}=1$.

of opposite spin. The most obvious one is the simultaneous jumps to the upper branch observed in Fig. $2 \mathrm{~d}$, showing that a population increase of the dominant polarization blueshifts the other one (see also Supplementary Information). This is in contrast to the common considerations that $\alpha_{2}<0$, used for the theoretical prediction $^{21}$. Repulsive interactions in the singlet configuration can originate from the proximity to the biexciton resonance ${ }^{26}$ and from the onset of exciton saturation.

A second important term of our model (see equation (3)) are the nonlinear losses. Experimental evidence for their influence is the narrowing of the power-dependent hysteresis under linearly polarized excitation. Their effect is most pronounced in Fig. 2c, where the hysteresis loop in $\sigma-$ is much reduced because of the large $\sigma+$ density. Moreover, in Fig. 2d,e, the $\sigma+$ population increases at the lower threshold of $\sigma-$. All of these features are qualitatively reproduced by our model (see Supplementary Information).

Physically, nonlinear losses are probably related to the formation of biexcitons when two polaritons of opposite spins collide. Detuning-dependent experiments revealed a clear enhancement of the nonlinear losses when the excitation energy is close to the biexciton resonance ${ }^{27}$ (see Supplementary Information). Our simulations have shown that nonlinear losses are responsible for the independence of the lower bistability thresholds. As we will see, this independence of the lower thresholds down to $\rho_{\text {pump }}=0$ is crucial for achieving polarization multistability.

In Fig. 3, we show the emission polarization $\rho_{\mathrm{C}}$ versus $\rho_{\text {pump }}$. The experimental data are taken in the same conditions as in Fig. 2. The indicated powers give the position of the system on the curves of Fig. 2. We see that, for a given $\rho_{\text {pump }}$ even three stable solutions for the $\rho_{\mathrm{C}}$ can be found (see Fig. 3b-d).

If both $\sigma+$ and $\sigma-$ polaritons are on their lower bistability branch, nonlinearities are negligible and $\rho_{\mathrm{C}}$ varies linearly with $\rho_{\text {pump }}$. Therefore, to observe multistability, we first initialize the system by using high excitation power $P_{\text {pump }}$ with $\rho_{\text {pump }}=1$ to place the $\sigma+$ polaritons on the upper branch. $P_{\text {pump }}$ is then decreased to the desired value, and $\rho_{\text {pump }}$ is scanned back and forth between +1 and -1 . Experimental data are shown in Fig. 3a-d.
At high excitation power (see Fig. 3a), by decreasing $\rho_{\text {pump }}$, we first observe a jump from $\rho_{\mathrm{C}}=1$ to $\rho_{\mathrm{C}}=0.4$ because the $\sigma-$ population becomes sufficient to jump to the upper branch. By going further, at $\rho_{\text {pump }}=-0.5$, the $\sigma+$ population becomes too low to stay on the upper branch and $\rho_{\mathrm{C}}$ jumps to -1 . The presence of hysteresis for both jumps is evidence of polarization bistability for each population.

A decrease of the excitation power causes the two hysteresis loops to open and to get closer to the centre of the plot (see Fig. 3b). The reason is that the decrease of excitation density must be compensated by the polarization degree for $\sigma$ - polaritons to jump to the upper branch (see equation (1)).

On a further decrease of the excitation power to the vicinity of the lower thresholds, the two polarization hysteresis loops merge, which demonstrates polarization multistability (see Fig. 3d).

Strikingly, at even lower excitation power, both $\sigma+$ and $\sigma-$ populations jump together in opposite directions without staying on the middle branch (Fig. $3 \mathrm{~d}, \mathrm{~g}$ ). The polarization degree switches abruptly from $\rho_{\mathrm{C}}>0.97$ to $\rho_{\mathrm{C}}<-0.97$. We observe a total spin flip of the $\sim 10^{2}$ polaritons present in the system, for a small variation of the excitation polarization degree $\left(\Delta \rho_{\text {pump }} \simeq 0.2\right)$. The system behaves under these conditions as a spin trigger, analogous to the Schmitt trigger in electronics. Theoretical simulations are shown in Fig. 3e-h. Our model is again in good qualitative agreement with the experimental results. Multistability and spin triggering are obtained in our simulations. Details on quantitative comparisons are given in the Supplementary Information.

In both spin multistability and spin triggering cases, three stable states exist for $\rho_{\mathrm{C}}$ under linearly polarized excitation: +1 , 0 and -1 . A fourth stable state actually exists because the $\rho_{\mathrm{C}}=0$ state is degenerate in intensity and can also be obtained if both populations are on their lower branch. In this case, any memory of high population regime is lost. The system is OFF, and has to be re-initialized to switch reversibly.

We verified the stability of the $\rho_{\mathrm{C}}=0$ solution by scanning the excitation polarization degree back and forth (see Fig. 4a). In the region of the multistability gap, a linear polarization branch 


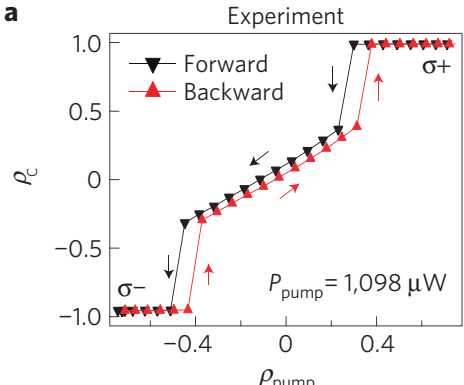

e

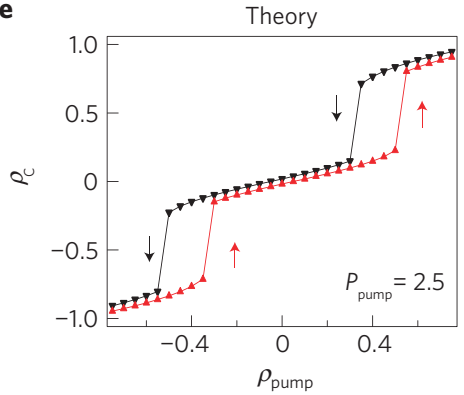

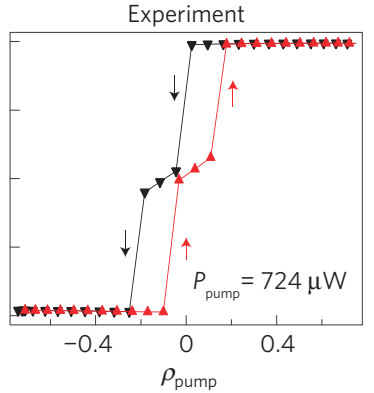

$\mathbf{f}$

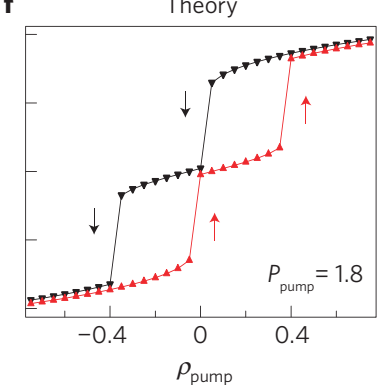

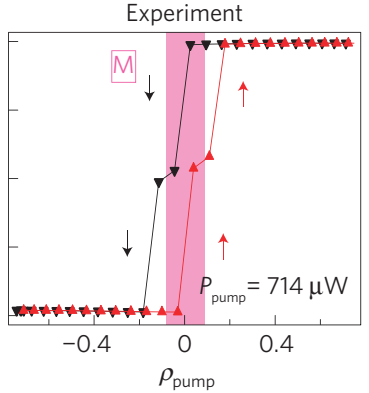

$\mathbf{g}$

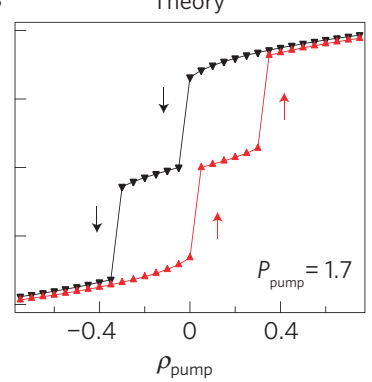

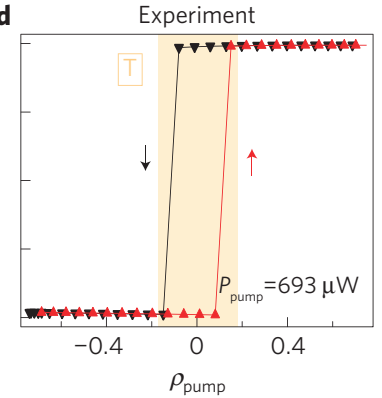

h

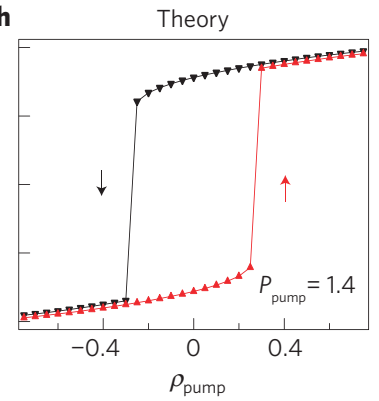

Figure 3 | Multistability. a-d, Experiments in the same conditions as in Fig. 2. The system is first initialized on the upper bistability branch of $\sigma+$ polaritons. Then, $\rho_{\text {pump }}$ is scanned between +1 and -1 , for different values of $P_{\text {pump }}$. a, At high excitation power, the scan reveals two distinct polarization hysteresis loops. $\mathbf{b}$, A decrease of $P_{\text {pump }}$ brings the two loops close to each other. $\mathbf{c}$, Multistability (M) occurs when the power is sufficiently decreased so that the two hystereses merge. In the $\mathrm{M}$ region, the system has three stable polarization states, for a given excitation condition. $\mathbf{d}$, Spin trigger ( $T$ ). At an even lower $P_{\text {pump }}$ the hystereses completely overlap. The whole system switches from spin-up to spin-down with a hysteresis in $\rho_{\text {pump }}$ of 0.2 . e-h, Our model reproduces the features of the experiments with good qualitative agreement.

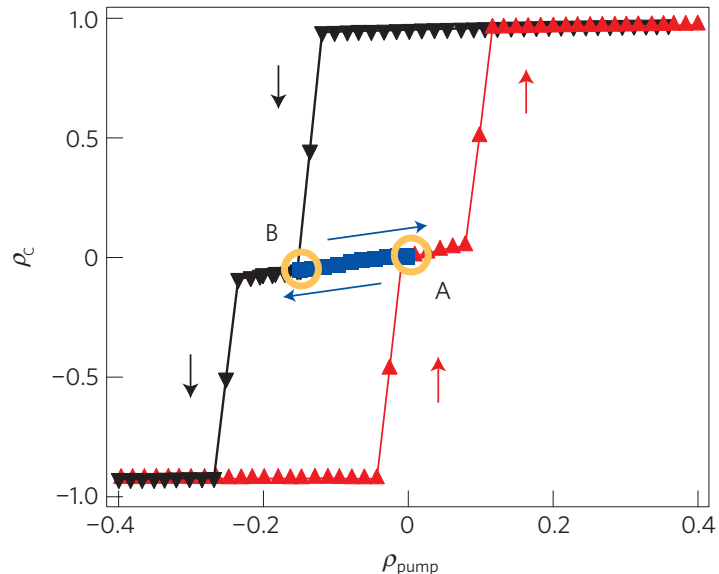

b

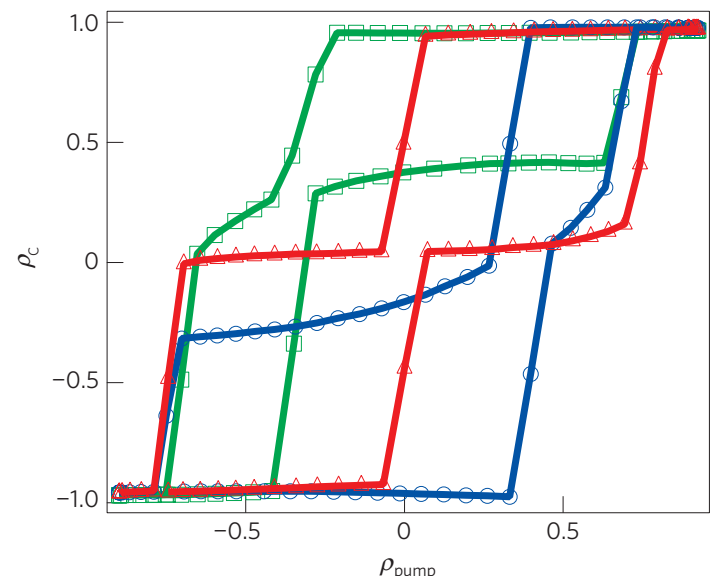

Figure 4 | Tuning the multistability. a, Stability. The middle branch of the multistability is stable and can be covered at will. We interrupted the scan of $\rho_{\text {pump }}$ at $\mathrm{A}$ and changed it forward and backward between A and B. This experiment shows that three stable solutions exist in the multistability region. b. Asymmetric multistability. By rotating the excitation linear axis, we tune the linear polarization splitting and modify the symmetry of the multistability cycle. We thus can displace the middle branch towards values of $\rho_{\mathrm{C}} \neq 0$ as well as the multistability gap. Data are taken for three different orientations of the linear axis. The symmetric multistability cycle (triangles) corresponds to an orientation close to a main linear polarization splitting axis. The two others correspond to rotations of $+40^{\circ}$ (circles) and $-40^{\circ}$ (squares).

is clearly evidenced. Moreover, because of the linear polarization splitting, the multistability region can be asymmetric with respect to $\rho_{\text {pump }}=0$, favouring one or the other spin orientation. The symmetry of the multistability cycle is tuned by rotating the linear polarization axis of the excitation beam (see the Methods section). The multistability gap can be displaced to regions of elliptical polarization (see Fig. 4b). The tunability of the multistability holds great promise for the development of spin-dependent optoelectronics devices.

To characterize the complete polariton spin state, we measure the three Stokes parameters (see Supplementary Information). We are then able to track the trajectory of the pseudospin vector in the Bloch sphere during the multistability cycle, as shown in Fig. 5. One easily identifies the features of multistability. The experiment starts at the $\sigma-$ pole of the sphere, where the pseudospin vector is pinned before jumping abruptly close to the $\mathrm{D}+$ state. A wide precession towards the $\mathrm{H}$ state is then observed before the second jump to the $\sigma+$ pole occurs (arrows). On the backward path, the third jump brings the pseudospin farther from the $\mathrm{D}+$ state, producing a gap in the precession trajectory (purple circle in Fig. 5). Then the pseudospin precesses towards the $\mathrm{V}$ state and finally jumps down to the $\sigma-$ state. 


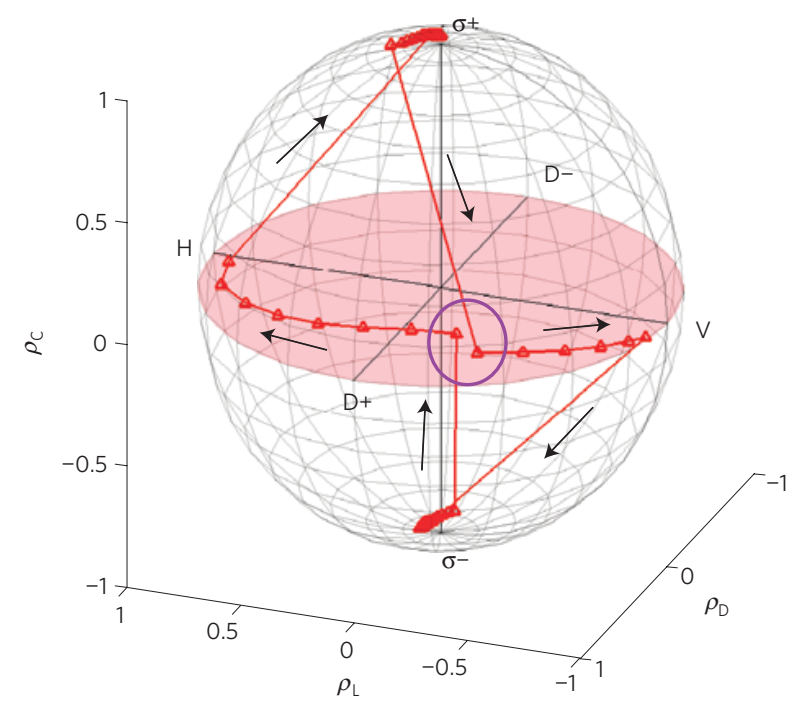

Figure $\mathbf{5}$ | Spin multistability. We measure the three Stokes parameters to reconstruct the trajectory of the pseudospin vector in the Bloch sphere. The four jumps and the gap (circle) in the middle branch trajectory are characteristic of the multistability of the polariton spin. The spin coherence is maintained during the whole cycle. The method for measuring the Stokes parameters is provided in the Supplementary Information, together with a comparison with theoretical simulations and a movie of the experiment.

During the multistability cycle, the trajectory of the polariton pseudospin vector strongly differs from the one of the excitation polarization vector (see Supplementary Information). The conservation of the pseudospin coherence during the multistability cycle demonstrates the coherent control of a macroscopic spin ensemble.

Our experiments demonstrate the multivalued switching of a spin ensemble in the solid state. The control and tunability of spin multistability clearly establish the feasibility of spintronic devices based on localized polaritons ${ }^{22,23}$. These results could easily be combined with polariton diode technologies ${ }^{13,14}$ to develop new integrated devices ${ }^{28}$. In addition, microcavities based on large-bandgap materials ${ }^{29}$ might be used for the development of devices operating at room temperature. Finally, our experimental procedure can be used to study spinor bistability and to realize spin multistability in other kinds of photonic system ${ }^{30-33}$.

\section{Methods}

Sample and set-up. The sample consists of a $\lambda$ GaAs/AlAs microcavity with a single 8-nm-thick $\operatorname{In}_{0.04} \mathrm{Ga}_{0.96}$ As quantum well. The top and bottom distributed Bragg reflectors are made of 21 and $22 \mathrm{GaAs} / \mathrm{AlAs} \lambda / 4$ layer pairs, respectively. On top of the cavity spacer, patterned mesas of $6 \mathrm{~nm}$ height act as traps for polaritons ${ }^{25}$. We measured a $3.35 \mathrm{meV}$ Rabi splitting in the mesas. A gradient in the sample thickness allows us to adjust the polariton energy $\delta^{\prime}$ by changing the position on the sample. Owing to confinement, we can excite single polariton states with well-defined energy. Typical linewidths are about $80 \mu \mathrm{eV}$ and the spacing between the first excited state and the ground state is close to $1 \mathrm{meV}$. We work in the transmission configuration. The sample is kept at $5 \mathrm{~K}$ in a helium-flow cryostat. On the excitation side, the circular polarization degree of the excitation is controlled using a quarter-wave plate. The linear axis of the excitation beam can be rotated with respect to the horizontal polarization axis using a half-wave plate. On the detection side, the emission is polarization-resolved using a second quarter-wave plate, followed by a polarization beam splitter. The signal is then sent to a high-dynamic-range charge-coupled device (CCD) camera.

Stabilization. Multivalued spin switching demands a very good stabilization of the experiment and a high accuracy in excitation power and polarization degree. To meet these requirements, we use a single-mode excitation laser and mount several optics elements on motorized stages. The experiment is fully automated and computer-controlled so that we have synchronization of the power or polarization scans with the polarization-resolved detection. The sample position is another crucial source of fluctuations. We control the position of the 3- $\mu \mathrm{m}$-diameter mesa in the centre $20-\mu \mathrm{m}$-diameter excitation spot with micrometre precision using piezoelectric actuators. We have $0.5 \%$ accuracy on the excitation power and a maximal $2 \%$ error on the emission intensity. The latter leads to a maximal error of \pm 0.05 on the polarization degree.

Tunability. We have full optical control of polarization multistability in our system. We control the overlap of the $\sigma+$ and $\sigma-$ polarization bistability curves by tuning the excitation power. The laser detuning controls the width of the polarization hysteresis. The smaller the laser detuning, the smaller the polarization hysteresis. Nonlinear losses are tuned by adjusting the polariton-exciton detuning $\delta^{\prime}$ and laser detuning $\Delta$ (see Supplementary Information). Finally the multistability region can be displaced by rotating the excitation linear axes with respect to the crystallographic axes of the sample. All of these parameters can be combined to design tunable devices with very different properties. In particular, we observed differences in the sensitivity of the spin trigger ranging over one order of magnitude (from 0.05 to 0.38 hysteresis width).

Linear polarization splitting We carried out high-precision measurements of the polarization-resolved transmission spectrum in $3 \mu \mathrm{m}$ mesas. The excitation linear polarization axis was rotated with respect to our reference horizontal polarization axis, with steps of $4^{\circ}$. For each orientation, we scanned the laser energy over $70 \mathrm{GHz}$ with steps of $0.5 \mathrm{GHz}$. We obtained a linear polarization splitting of about $20 \mu \mathrm{eV}$, with orthogonal axes at $\pm 45^{\circ}$ with respect to our reference horizontal polarization axis. These axes correspond to the [001] and [010] crystallographic axes and are not related to any ellipticity of the mesas.

\section{Received 9 February 2010; accepted 22 May 2010;} published online 4 July 2010

\section{References}

1. Myers, R. C. et al. Zero-field optical manipulation of magnetic ions in semiconductors. Nature Mater. 7, 203-208 (2008).

2. Imamoğlu, A. Cavity QED based on collective magnetic dipole coupling: Spin ensembles as hybrid Two-Level systems. Phys. Rev. Lett. 102, 083602 (2009).

3. Greilich, A. et al. Ultrafast optical rotations of electron spins in quantum dots. Nature Phys. 5, 262-266 (2009).

4. Awschalom, D. D. \& Flatte, M. E. Challenges for semiconductor spintronics. Nature Phys. 3, 153-159 (2007).

5. Lagoudakis, P. G. et al. Stimulated spin dynamics of polaritons in semiconductor microcavities. Phys. Rev. B 65, 161310 (2002).

6. Martín, M. D., Aichmayr, G., Viña, L. \& André, R. Polarization control of the nonlinear emission of semiconductor microcavities. Phys. Rev. Lett. 89, 077402 (2002).

7. Kasprzak, J. et al. Bose-Einstein condensation of exciton polaritons. Nature 443, 409-414 (2006).

8. Bajoni, D. et al. Polariton laser using single micropillar GaAs-GaAlAs semiconductor cavities. Phys. Rev. Lett. 100, 047401 (2008).

9. Amo, A. et al. Collective fluid dynamics of a polariton condensate in a semiconductor microcavity. Nature 457, 291-295 (2009).

10. Leyder, C. et al. Observation of the optical spin Hall effect. Nature Phys. 3, 628-631 (2007).

11. Lagoudakis, K. G. et al. Observation of half-quantum vortices in an exciton-polariton condensate. Science 326, 974-976 (2009).

12. Baas, A., Karr, J. P., Eleuch, H. \& Giacobino, E. Optical bistability in semiconductor microcavities. Phys. Rev. A 69, 023809 (2004).

13. Bajoni, D. et al. Optical bistability in a GaAs-based polariton diode. Phys. Rev. Lett. 101, 266402 (2008).

14. Tsintzos, S. I., Pelekanos, N. T., Konstantinidis, G., Hatzopoulos, Z. \& Savvidis, P. G. A GaAs polariton light-emitting diode operating near room temperature. Nature 453, 372-375 (2008).

15. Deveaud-Pledran, B. Solid-state physics: Polaritronics in view. Nature 453, 297-298 (2008).

16. Shelykh, I. et al. Semiconductor microcavity as a spin-dependent optoelectronic device. Phys. Rev. B 70, 035320 (2004).

17. Kitano, M., Yabuzaki, T. \& Ogawa, T. Optical tristability. Phys. Rev. Lett. 46, 926-929 (1981).

18. Cecchi, S., Giusfredi, G., Petriella, E. \& Salieri, P. Observation of optical tristability in sodium vapors. Phys. Rev. Lett. 49, 1928-1931 (1982).

19. Dery, H., Dalal, P., Cywinski, L. \& Sham, L. J. Spin-based logic in semiconductors for reconfigurable large-scale circuits. Nature 447, 573-576 (2007).

20. Behin-Aein, B., Datta, D., Salahuddin, S. \& Datta, S. Proposal for an all-spin logic device with built-in memory. Nature Nanotech. 5, 266-270 (2010).

21. Gippius, N. A. et al. Polarization multistability of cavity polaritons. Phys. Rev. Lett. 98, 236401 (2007).

22. Shelykh, I. A., Liew, T. C. H. \& Kavokin, A. V. Spin rings in semiconductor microcavities. Phys. Rev. Lett. 100, 116401 (2008).

23. Liew, T. C. H., Kavokin, A. V. \& Shelykh, I. A. Optical circuits based on polariton neurons in semiconductor microcavities. Phys. Rev. Lett. 101, 016402 (2008). 
24. Amo, A. et al. Exciton-polariton spin switches. Nature Photon. 4, 361-366 (2010).

25. El-Daï, O. et al. Polariton quantum boxes in semiconductor microcavities. Appl. Phys. Lett. 88, 061105 (2006).

26. Kwong, N. H., Takayama, R., Rumyantsev, I., Kuwata-Gonokami, M. \& Binder, R. Third-order exciton-correlation and nonlinear cavity-polariton effects in semiconductor microcavities. Phys. Rev. B 64, 045316 (2001).

27. Saba, M. et al. Crossover from exciton to biexciton polaritons in semiconductor microcavities. Phys. Rev. Lett. 85, 385 (2000).

28. Ramkumar, K. \& Nagaraj, K. A ternary schmitt trigger. IEEE Trans, Circuits Systems 32, 732-735 (1985).

29. Christmann, G., Butté, R., Feltin, E., Carlin, J-F. \& Grandjean, N. Room temperature polariton lasing in a GaN/AlGaN multiple quantum well microcavity. Appl. Phys. Lett. 93, 051102 (2008).

30. Jonsson, F. \& Flytzanis, C. Polarization state controlled multistability of a nonlinear magneto-optic cavity. Phys. Rev. Lett. 82, 1426 (1999).

31. SoljaCiC, M. \& Joannopoulos, J. D. Enhancement of nonlinear effects using photonic crystals. Nature Mater. 3, 211-219 (2004)

32. Zhou, L., Pu, H., Ling, H. Y. \& Zhang, W. Cavity-mediated strong matter wave bistability in a spin-1 condensate. Phys. Rev. Lett. 103, 160403 (2009).
33. O’Brien, J. L., Furusawa, A. \& Vuckovic, J. Photonic quantum technologies. Nature Photon. 3, 687-695 (2009).

\section{Acknowledgements}

The authors thank D. Sarchi, I. Carusotto, M. Portella-Oberli, T. C. H. Liew and V. Savona for fruitful discussions, and O. El Daiff for his contributions in engineering the sample. The present work was supported by the Swiss National Research Foundation through 'NCCR Quantum Photonics'.

\section{Author contributions}

T.K.P. carried out the experiments and data analysis. M.W. developed the theoretical model. M.W. and Y.L. did the simulations. F.M.G. made the sample. B.D.P. supervised the project.

\section{Additional information}

The authors declare no competing financial interests. Supplementary information accompanies this paper on www.nature.com/naturematerials. Reprints and permissions information is available online at http://npg.nature.com/reprintsandpermissions. Correspondence and requests for materials should be addressed to T.K.P. 